\title{
Suppression method of the condensation reaction during phenol liquefaction of woody material
}

\author{
Q. Wang ${ }^{1}$, N. Mitsumura ${ }^{1}$, Q. Chen ${ }^{1}$, P. Apar ${ }^{1}$, H. Niida ${ }^{1}$, S. Ito ${ }^{1}$, \\ T. Endo ${ }^{1}$, S. Animesh ${ }^{1}$, H. Kurokawa ${ }^{1}$, K. Sekiguchi ${ }^{1}$ \\ \& K. Sugiyama ${ }^{2}$ \\ ${ }^{1}$ Graduate School of Science and Engineering, Saitama University, Japan \\ ${ }^{2}$ Hachinohe National College of Technology, Japan
}

\begin{abstract}
The liquefaction of woody materials in the presence of phenol and acid catalyst is a promising method to utilize the waste woody materials into phenolic resin. However, the condensation reaction is a major problem for its practical applications. In order to suppress condensation reactions, methanol was added to the liquefaction medium. Even the intense condensation reactions were suppressed by the addition of $50 \%$ methanol ( $\mathrm{mol} \%$ to phenol). The effect of methanol was further confirmed by the measurement of molecular weight distribution. In the case of mild condensation, addition of 5\% methanol suppressed the production of the residues. At the same time, the liquefaction rates overall were faster than the case without methanol. It was inferred that the existence of methanol lowered the amount of combined phenol which could be the reaction site of the condensation reaction. On the other hand, larger amounts of methanol $(100 \%)$ retarded the liquefaction rate and the consumption rate of phenol. The actual reaction temperature in the reactor was lower than the setup temperature of the oil bath when the methanol was added. The investigation of the IR spectra showed that there were almost no differences between the functional groups of the liquefied products obtained with and without methanol. The addition of small amounts of methanol could be applicable because the disadvantages of methanol addition could be reduced.

Keywords: waste woody materials, liquefaction, phenol, condensation reaction, suppression method, methanol.
\end{abstract}




\section{Introduction}

Woody materials are one of the most abundant biomass resources around the world. Most parts of them are used as pulp or solid fuel. On the other hand, some kinds of waste woody materials are hardly used at all. For example, 99\% of 8 million tons of forest residues, such as forest thinned waste materials, are not utilized in Japan [1]. These waste woody materials should be utilized instead of fossil resources in order to prevent global warming. However, utilization of woody materials is rather difficult because of their components such as cellulose and lignin which have very hard and complex structures.

Based on that background, increasing interest is dedicated to conversion processes among which liquefaction can be of interest. This is a solvolysis method aimed at converting all of the woody constituents into a liquid matrix (known as 'liquefied wood') through a reaction involving a solvent (in this case phenol) and an acid catalyst (such as sulfuric acid) at temperatures varying from 100 to $200^{\circ} \mathrm{C}$ without any chemical pretreatments. During the liquefaction process, woody constituents are degraded to smaller molecules which may have reactivity comparable to phenol. Liquefied wood can be used as raw materials to produce phenolic resin which may be of interest for the plastics industry. Some research studies into making various kinds of phenolic resins such as moldings [2], foams [3] and fibers [4] from liquefied wood have been reported.

However, popularizing this technique is a big problem because of the condensation reaction during the liquefaction. It is a spontaneously-occurring reaction which causes polymerization of the molecules of liquefied woody materials [5]. The condensation reaction can be regarded as an opposite reaction of liquefaction which degrades the molecules of liquefied wood. This reaction does not necessarily occur but can occur under some reaction conditions for various reasons [5]. This reaction has an adverse effect on the synthesis of phenolic resin. In our previous studies, the effects of various reaction conditions on condensation were investigated [6].

To inhibit the condensation reaction, high phenol concentrations against woody materials are needed to be added to the liquefaction system [7]. However, the consumption of phenol has to be limited because it is made from exhaustible petroleum resources. Therefore, it is considered more efficient to suppress the condensation reaction with low additional amounts of phenol. In this study, we tried to achieve this by the addition of methanol to the liquefaction reaction. Moreover, the effect of methanol addition on the liquefaction product was investigated.

\section{Experimental procedures}

\subsection{Materials}

Woody meals (or sawdust) of Japanese cedar (Cryptomeria japonica) were used as raw waste woody materials for the liquefaction. These waste woody materials were collected from the waste disposal plant of Saitama, Japan, and then sieved 
into given particle sizes $(<0.250 \mathrm{~mm})$ and dried at room temperature for 12 hours before the liquefaction experiment. Sulfuric acid was used as a mineral acid catalyst.

\subsection{Methods}

\subsubsection{Liquefaction of woody materials}

The liquefaction was carried out using $500 \mathrm{~mL}$ three necked flask equipped with a mechanical stirrer and reflux cooler (Figure 1). An oil bath was used to heat the flask. All reaction conditions performed in this study are shown in Table 1. The additional amount of methanol was setup as mol\% against phenol. The given amounts of phenol, sulfuric acid and methanol (if needed) were charged into the flask and mixed well. Then woody meal was added. The time at which the flask was immersed into the oil bath after the addition of woody meal was regarded as start time of reaction. The reaction was run up to 300 minutes. The samples were taken from the flask several times during the period and put into vials. Then it was immersed in cold water to stop the reaction.

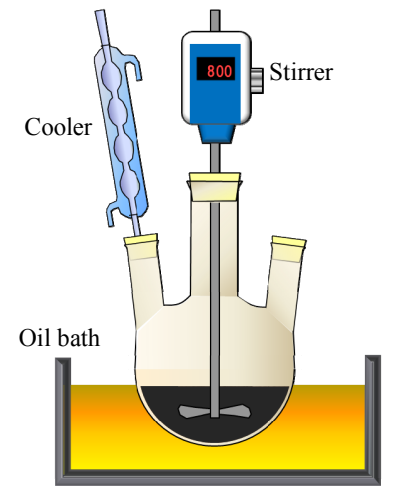

Figure 1: Reactor of liquefaction.

Table 1: $\quad$ Reaction conditions.

\begin{tabular}{cccccc}
\hline & $\begin{array}{c}\text { Methanol } \\
(\mathrm{mmol})\end{array}$ & $\begin{array}{c}\text { Phenol } \\
(\mathrm{mmol})\end{array}$ & $\begin{array}{c}\text { Woody } \\
\text { meals }(\mathrm{g})\end{array}$ & $\begin{array}{c}\text { Sulfuric acid } \\
(\mathrm{mmol})\end{array}$ & $\begin{array}{c}\text { Reaction } \\
\text { temp. }\left({ }^{\circ} \mathrm{C}\right)\end{array}$ \\
\hline Run 1 & - & 355 & 16.7 & 69.8 & 180 \\
Run 2 & $178(50 \%)$ & 355 & 16.7 & 69.8 & 180 \\
Run 3 & - & 355 & 16.7 & 69.8 & 160 \\
Run 4 & $355(100 \%)$ & 355 & 16.7 & 69.8 & 160 \\
Run 5 & $53.2(15 \%)$ & 355 & 16.7 & 69.8 & 160 \\
Run 6 & $17.8(5 \%)$ & 355 & 16.7 & 69.8 & 160 \\
Run 7 & - & 484 & 4.55 & 14.2 & 160 \\
Run 8 & $242(50 \%)$ & 484 & 4.55 & 14.2 & 160 \\
\hline
\end{tabular}

*1: Percentage shown in parentheses is mol\% against phenol 


\subsubsection{Measurement of residue contents}

The outcome of the reactions including the condensation reaction were evaluated by measuring the residue content. The residues consist of solid mixed matter of the unliquefied woody meal and/or the condensed matters which polymerized to be a solid substance. It is known that the residue amount decreases due to the liquefaction at earlier stages of the reaction, while it increases at later stages due to the condensation reaction [8]. The increase rate of the residue amount reflects the extent of the condensation reaction. In order to measure the residue content, the samples were diluted with excess amounts of methanol and filtrated using glass fibre filters (GA-100, Toyo Roshi Kaisha, Co., Ltd.) under reduced pressure washing with methanol. The residues were dried in an oven at $105^{\circ} \mathrm{C}$ for 24 hours and then weighed. The residue content in the samples was calculated using the following equation (1):

$$
\text { Residue content }(\%)=R / R_{0} \times 100
$$

Here, $R$ is the weight ratio of the residue in the sample and $R_{0}$ the weight ratio of the woody meals in the raw materials.

\subsubsection{Measurement of phenol content}

The phenol content in the samples was measured by using a high performance liquid chromatograph (HPLC) equipped with a detector of SPD-10A (Shimadzu Co. Ltd.), and a column of Wakosil-PAHs (Wako Co., Ltd., Japan). The flow rate, the oven temperature and detection wavelength were $2 \mathrm{ml} / \mathrm{min}, 40^{\circ} \mathrm{C}$ and $272 \mathrm{~nm}$ respectively. Methanol/ $\mathrm{H}_{2} \mathrm{O}(1: 2, \mathrm{v} / \mathrm{v})$ mixed solvent was used as a mobile phase. The sample was diluted by $0.1 \mathrm{wt} . \%$ by methanol/water $(4: 1, \mathrm{v} / \mathrm{v})$ mixed solvent for the measurement. A syringe filter $(0.45 \mu \mathrm{m}$, Toyo Roshi Kaisha Co., Ltd., Japan) was used to remove the residue in the sample when the sample was injected. The phenol content in the samples was calculated using the following equation (2):

Phenol content $(\%)=P / P_{0} \times 100$

Here, $P$ is the weight ratio of the phenol in the sample and $P_{0}$ the weight ratio of phenol in the raw materials.

\subsubsection{Measurement of molecular weight distribution}

Measurement of the molecular weight distributions of liquefied products were performed by a Gel permeation chromatograph (GPC) equipped with a differential refractive index detector (Shimadzu Co., Ltd., Japan). The columns used were KF-802 (Showa denko Co., Ltd., Japan). Tetrahydrofuran was used as a mobile phase. Flow rate was $1 \mathrm{ml} / \mathrm{min}$. The other methods were similar to the measurement of phenol concentration. The detector response was plotted against retention time to show the molecular weight distribution.

\subsubsection{Measurement of chemical structure}

The liquefied products (except the residue) were analyzed by using FT-IR spectrometer (Model IR-6100, Jasco Co., Ltd., Japan). After removing the residue from the samples by filtration with methanol, methanol was removed from the filtrates by using rotary-evaporation. The obtained liquefied products were mixed with $\mathrm{KBr}$ powder (1:200) using the $\mathrm{KBr}$ pellet method. 


\section{Results and discussions}

\subsection{Confirmation of the effect of methanol addition on the liquefaction system}

The effect of methanol addition to the liquefaction system is shown in Figure 2. In the case of Run 1 (where methanol was not added), the residue content decreased to $8.7 \%$ at $30 \mathrm{~min}$. After that, the residue content increased drastically and reached $67 \%$ at $300 \mathrm{~min}$. This indicated that an intense condensation reaction occurred. On the other hand, in the case of Run 2 where $50 \%$ methanol was added, the residue content was $10 \%$ at $30 \mathrm{~min}$. It decreased gradually until $180 \mathrm{~min}$ and increased slightly at $300 \mathrm{~min}$. The final value was $11 \%$, indicating the condensation reaction was moderate compared to Run 1 due to addition of methanol.

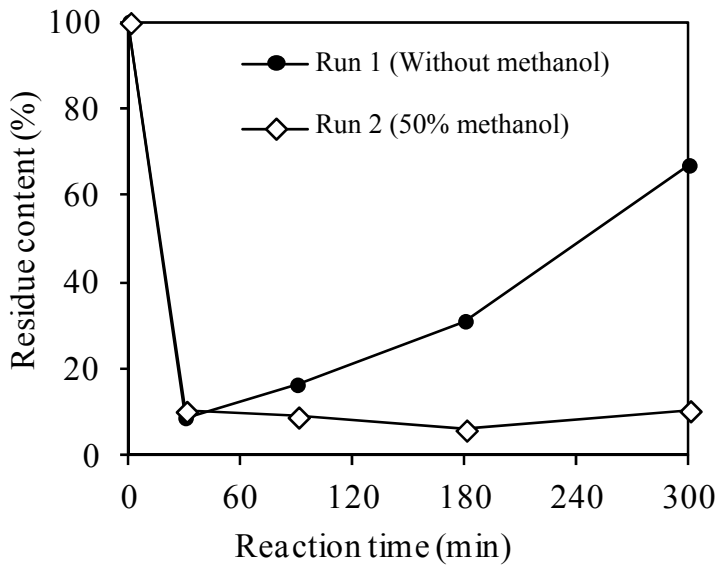

Figure 2: Time course of residue content for Run 1 and 2. Reaction conditions: phenol: $355 \mathrm{mmol}$, woody meal: $16.7 \mathrm{~g}$, sulfuric acid: $69.8 \mathrm{mmol}$, reaction temperature: $180^{\circ} \mathrm{C}$.

Measurement of the residue content can evaluate only the "solid" condensed matter. In order to confirm the further effects of methanol on the condensation, the molecular weight distribution in the "liquid phase" was measured using GPC for each reaction conditions (Figures 3(a) and (b)). In the figures, there were two sharp peaks at both sides of the chromatogram. The sharp peak was derived mainly from unreacted phenol appearing after $9 \mathrm{~min}$. The other sharp peak appearing after $5 \mathrm{~min}$ is derived from a higher molecule than the exclusion limit of the GPC column, which was mainly due to the condensed matter. First, in the case of Run 2, it was clearly observed that the sharp peak of high molecular weight increased during 30-180 min of the reaction time while it decreased during 180-300 min. In general, the peak of high molecular weight increases when the condensation occurred. However, if the condensation proceeds further, the peak of high molecular weight diminishes because the liquid condensed 
matter becomes solid substance [5]. The peaks of higher molecules at 180 and 300 min of reaction time in Run 1 were shorter than for Run 2. This was considered because the peak had already begun to diminish before $180 \mathrm{~min}$ of reaction time. Therefore, these results also showed that the condensation reaction was suppressed by the addition of methanol.
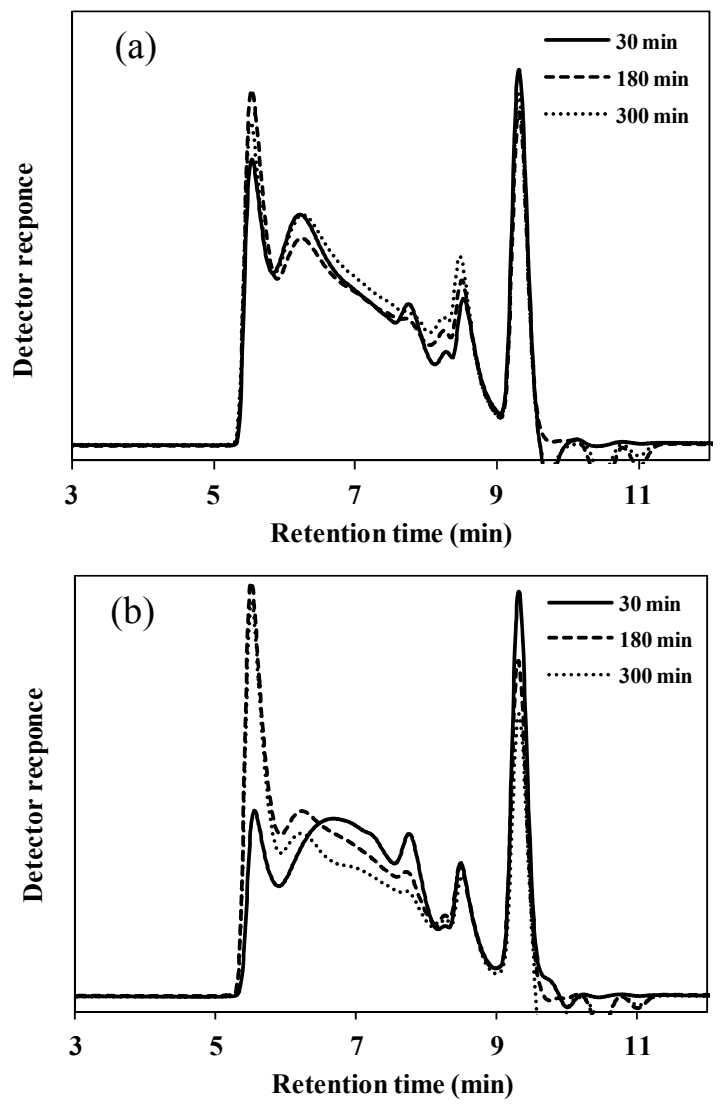

Figure 3: $\quad$ Molecular weight distribution for Run 1 (a) and Run 2 (b). Reaction conditions are phenol: $355 \mathrm{mmol}$, woody meal: $16.7 \mathrm{~g}$, sulfuric acid: $69.8 \mathrm{mmol}$, reaction temperature: $180^{\circ} \mathrm{C}$.

The addition of $50 \%$ methanol seemed to be too high. The condensation reaction was not suppressed completely even with the addition of such high amounts of methanol. Here the liquefaction was performed under reaction conditions where the condensation reaction became so remarkable. This condition was not thought to be applicable. Therefore, the amount of methanol can be reduced further under more applicable reaction conditions with mild condensation reaction. So, the effect of additional amounts was investigated in the next section. 


\subsection{Effect of additional amounts of methanol on the condensation reaction}

The effect of additional amounts of methanol was investigated under more applicable reaction conditions where the mild condensation reaction occurred (Figure 4). In the case of Run 3 (where methanol was not added), the residue content decreased to $16 \%$ at $90 \mathrm{~min}$. but it slightly increased to $21 \%$ at $300 \mathrm{~min}$, indicating mild condensation occurred. When 100\% methanol was added (Run 4 ), it was observed that residue content was reduced although the condensation was suppressed. In the case of Run 6 (where 5\% methanol was added), the residue content was lower than Run 3 and the increase of residue content was almost suppressed. In the case of Run 5 where $15 \%$ methanol was added, a medium result was seen.

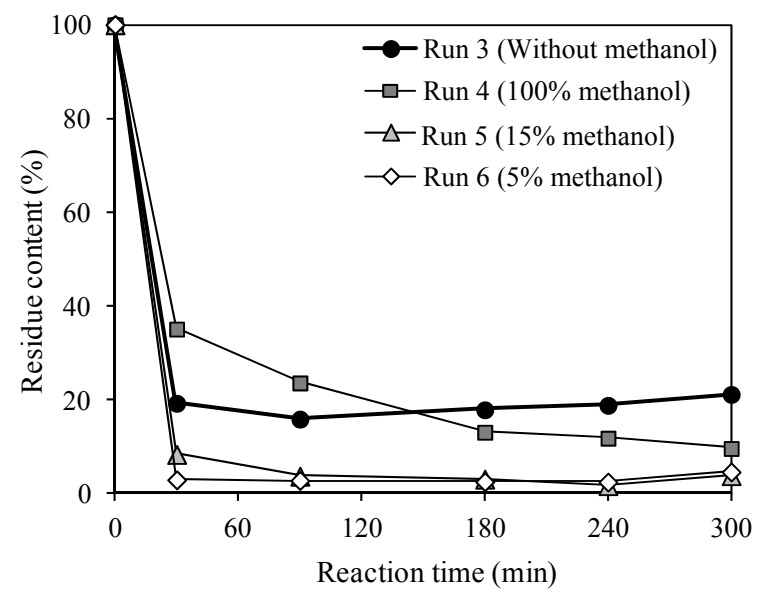

Figure 4: Time course of residue content for Run 3 Run 6. Reaction conditions are phenol: $355 \mathrm{mmol}$, woody meal: $16.7 \mathrm{~g}$, sulfuric acid: $69.8 \mathrm{mmol}$, reaction temperature: $160^{\circ} \mathrm{C}$.

The phenol concentration was measured using HPLC in Runs 3, 5 and 6 (Figure 5). It was clearly seen that the rate of phenol consumption was reduced when $100 \%$ methanol was added, compared to Run 3 (where methanol was not added). Therefore, it was found that too much addition of methanol inhibited the reaction of phenol and this would be responsible for the lowering of the decrease rate of the residue content in Figure 5. In the synthesis of the phenolic resin, "combined phenol" in the structure of liquefied woody constituents becomes the reaction site of polymerization [3]. Therefore phenol needs to react with woody constituents in the liquefaction. On the other hand, when 5\% methanol was added, the phenol content was almost the same as Run 3. From the above results, it can be concluded that small additional amounts of methanol can suppress the condensation reaction without inhibiting the reaction of phenol. 


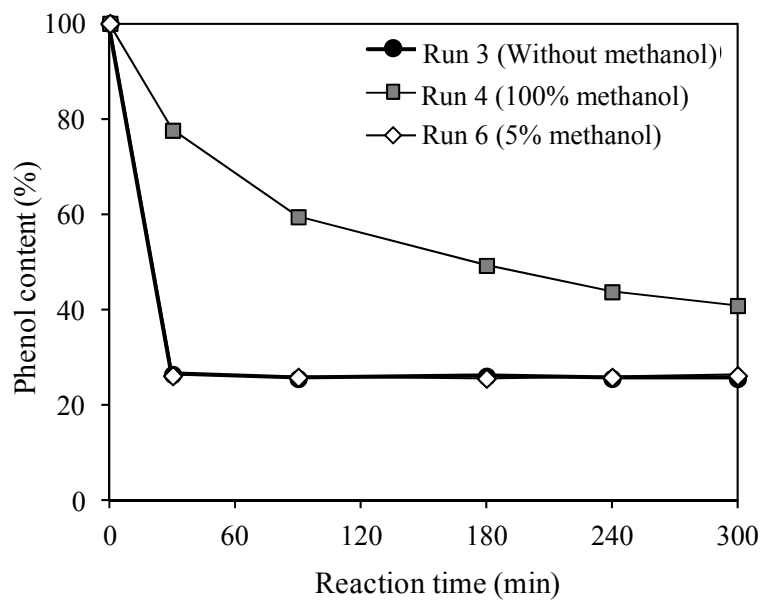

Figure 5: Time course of phenol content for Run 3, Run 4 and Run 6. Reaction conditions are phenol: 355 mmol, woody meal: $16.7 \mathrm{~g}$, sulfuric acid: $69.8 \mathrm{mmol}$, reaction temperature: $160^{\circ} \mathrm{C}$.

The liquefaction of wood with phenol can be regarded as "phenolysis". In the liquefaction, the woody constituent is degraded ionically and phenol reacts with it $[9,10]$. Then phenol becomes the combined phenol [9-12]. The combined phenol can be the reaction site of the condensation reaction [7]. On the other hand, methanol also reacts with cellulose (a major component of wood) under acidic conditions in a similar way to the phenolysis [13]. This reaction can be regarded as "methanolysis". Therefore, both the phenolysis and methanolysis occurred when the methanol was added to the liquefaction. As a result, the amount of the combined phenol decreased. This seemed to be responsible for the suppression of the condensation reaction.

\subsection{Effect of methanol addition on the liquefaction products}

The differences of liquefaction products when methanol was added were investigated. These experiments were performed under the reaction conditions where the condensation reaction hardly occurs because we needed to analyze the part of the products which are used to synthesize the phenolic resin. The condensed matter was not contained within it.

Here the reactions were performed under phenol rich conditions (10 times the weight of woody meals). The time course of the residue content is shown in Figure 6. It was observed that residue content reduced to $6.3 \%$ at $30 \mathrm{~min}$ and then become almost stable when methanol was not added. On the other hand, in the case of Run 8 where $50 \%$ methanol was added, the residue content decreased gradually and became $4.7 \%$ at the end of the reaction. It was found that much methanol addition decreased the liquefaction rate which is also seen in Figure 5. This might be because of the lowering of the actual reaction temperature. 


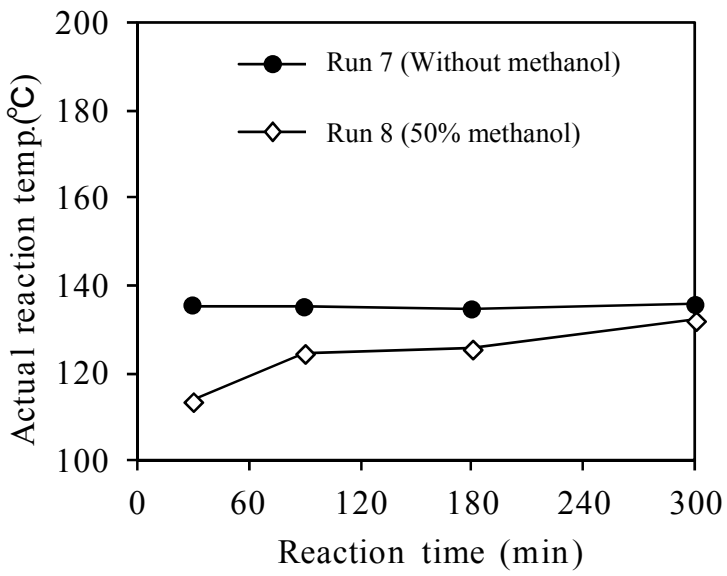

Figure 6: $\quad$ Time course of actual reaction temperature for Run 7 and Run 8 . Reaction conditions are phenol: $484 \mathrm{mmol}$, woody meal: $4.55 \mathrm{~g}$, sulphuric acid: $14.2 \mathrm{mmol}$, reaction temperature: $160^{\circ} \mathrm{C}$.

Although the setup temperature of the oil bath was $160^{\circ} \mathrm{C}$, the actual temperature in the reactor was lower than this in both cases (Figure 7). This is involved in the boiling point of the solvent. The boiling points of phenol and methanol are $182^{\circ} \mathrm{C}$ and $65^{\circ} \mathrm{C}$, respectively. Moreover, dehydration partly occurs in the liquefaction and water whose boiling point is $100^{\circ} \mathrm{C}$ is produced $[9,10]$. These compounds do not exceed the temperature in the liquid phase. In the case of Run 8 , the actual temperature was lower than in Run 7 because of the presence of methanol in addition to water. Because methanol was consumed in methanolysis, the actual temperature gradually increased. On the other hand, in Run 7, the actual temperature was almost stable. This was considered because the hydrolysis occurred in addition to the dehydration and the total amount of water did not change. At the first stage of the reaction, the actual difference in temperature was high between Runs 7 and 8 . That might be why the reaction rate of Run 8 was lower than for Run 7.

The samples taken at $300 \mathrm{~min}$ were analyzed by using FT-IR (Figure 8). It was shown that both spectra were almost homologous for their pattern. This indicated that these products had almost the same functional groups. More detailed analysis about the structure should be performed. On the other hand, the residues resulting from Run 7 where 50\% methanol was added were grey in color although the usual residue was pale or dark brown in color. The grey residue began to be seen from $180 \mathrm{~min}$ and its apparent amount increased at 300 min. Therefore, the grey residue was considered to be a product resulting from the reaction of methanol. From these observations, it was suggested that the products resulted from the reaction of methanol existed as residue which did not dissolve in methanol in filtration. Still it is not really clear whether the grey residues have reactivity for phenol resin synthesis. Therefore, we plan to investigate the structure of the residue. 


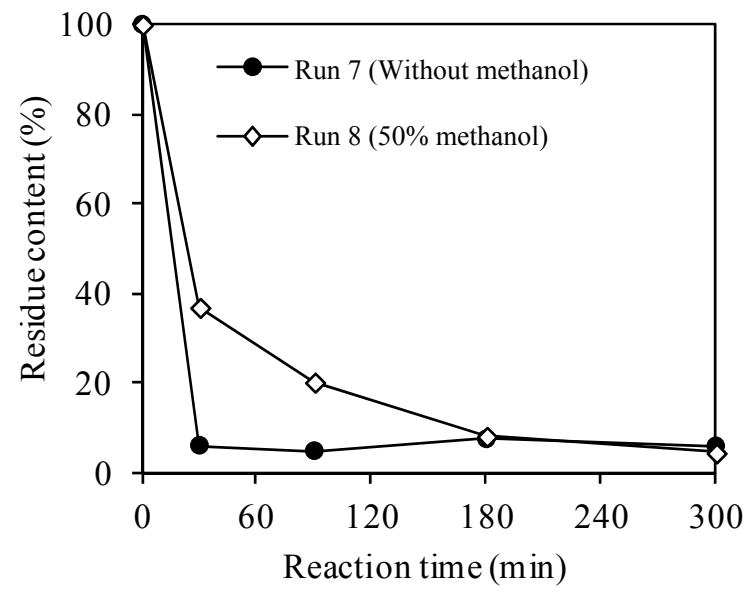

Figure 7: $\quad$ Time course of residue content for Run 7 and Run 8. Reaction conditions are phenol: $484 \mathrm{mmol}$, woody meal: $4.55 \mathrm{~g}$, sulphuric acid: $14.2 \mathrm{mmol}$, reaction temperature: $160^{\circ} \mathrm{C}$.

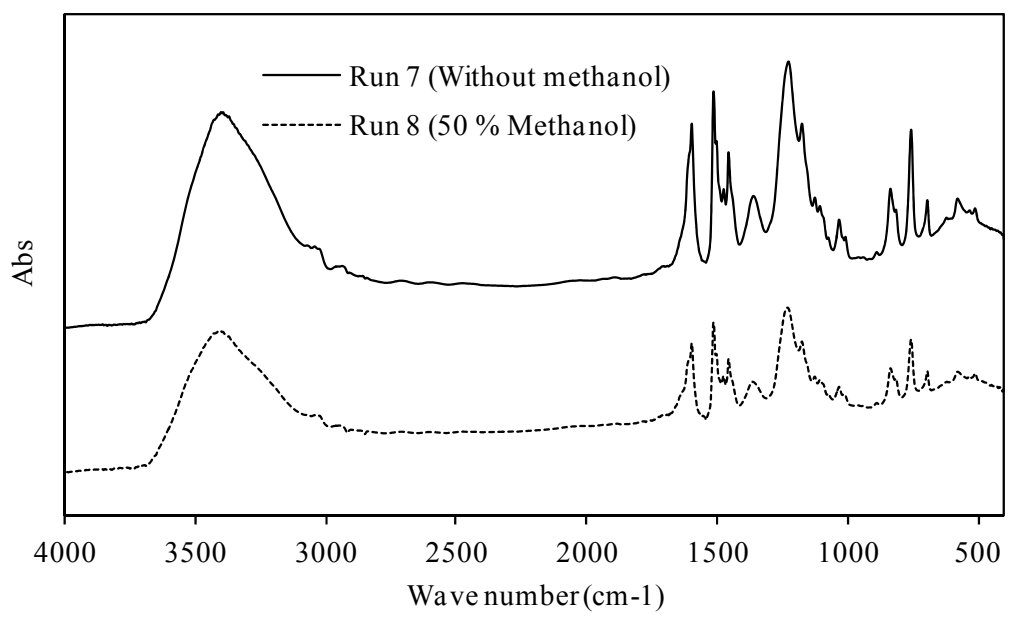

Figure 8: $\quad$ FT-IR spectra for liquefied products at 300 min for Run 7 and Run 8. Reaction conditions are phenol: 484 mmol, woody meal: $4.55 \mathrm{~g}$, sulphuric acid: $14.2 \mathrm{mmol}$, reaction temperature: $160^{\circ} \mathrm{C}$.

\section{Conclusions}

In our study, the effects of methanol addition on the condensation reaction during the phenol liquefaction of waste woody materials were investigated. It was shown that the addition of methanol suppressed the condensation reaction. 
Addition of too much methanol decreased the liquefaction rate. Small amounts suppressed the condensation reaction without retarding the liquefaction rate. The actual temperature in the reactor was lower than the setup temperature due to the presence of methanol and water. That might be the reason for lowering the liquefaction rate by the addition of methanol. The analysis using FT-IR spectrometry showed that the products resulting from the liquefaction with and without methanol had homologous structures. Grey residue existed in the product resulting from the liquefaction with methanol. These results indicated that there are two weak points of the addition of methanol. One is reducing the liquefaction rate by the lowering of the actual temperature. The other is production of a byproduct derived from methanolysis of the woody constituent. These weak points could be reduced by lowering the additional amount of methanol. Actually, the addition of 5\% methanol suppressed the condensation reaction remarkably without reducing the liquefaction rate. From our study, it was suggested that the addition of small amounts of methanol to the liquefaction was an effective way to suppress the condensation reaction.

\section{Acknowledgements}

Some works of this study were supported by the special funds for Basic Research (B) (No. 22404022, FY2010 2012) of a Grant-in-Aid for Scientific Research of the Japanese Ministry of Education, Culture, Sports, Science and Technology (MEXT), Japan.

\section{References}

[1] Ministry of Environment, Government of Japan, http://www.env.go.jp/ council/06earth/y0613-16/ref06-20.pdf (in Japanese).

[2] G. Li, T. Qin, Novolak type phenol formaldehyde resin from Waste brownrotted wood, Advanced Material Research, 217-218, 490-494, 2011.

[3] S. H. Lee, Y. Teramoto, N. Shiraishi, Resol-type phenolic resin from liquefied phenolated wood and its application to phenolic foam, Journal of Applied Polymer Science, 84, 468-472, 2001.

[4] X. Ma, G. Zhao, Structure and performance of fibers prepared from liquefied wood in phenol, Fibers and Polymers, 9, 405-409, 2008.

[5] M. Kobayashi, T. Asano, M. Kajiyama, B. Tomita, Analysis on residue formation during wood liquefaction with polyhydric alcohol, Journal of Wood Science, 50, 407-414, 2004.

[6] Q. Wang, N. Mitsumura, Q. Chen, P. Apar, Q. Qian, L. Gui, H. Niida, H. Kurokawa, K. Sekiguchi and Sugiyama K., Effect of the phenol concentrations on the condensation reaction during the liquefaction of waste woody materials with phenol, Waste Management and the Environment VI, Ecology and The Environment, 163, 355-366, 2012. (Paper Doi: 10.2495/WM120321). 
[7] Y. Zhang, A. Ikeda, N. Hori, A. Takemura, H. Ono, T. Yamada, Characterization of liquefied product from cellulose with phenol in the presence of sulfuric acid, Bioresource, 97, 313-321, 2006.

[8] M. Niu, G, Zhao, M. H.Alma, Polycondensation reaction and its mechanism during lignocellulosic liquefaction by an acid catalyst: a review, Forestry Studies in China 13, 71-79, 2011.

[9] Lin, L., Yao, Y., Yoshioka M., Shiraishi, N., Liquefaction mechanism of cellulose in the presence of phenol under acid catalysis, Carbohydrate polymers, 57, 123-129, 2004.

[10] L. Lin, Y. Yao, N. Shiraishi, Liquefaction mechanism of $\beta-O-4$ lignin model compound in the presence of phenol under acid catalysis. Part 2.Rreaction behavior and pathway, Holzforschung, 55, 625-630, 2001.

[11] S. P. Mun, J. P. Jang, Liquefaction of cellulose in the presence of phenol using $p$-toluene sulfonic acid as a catalyst, Journal of Industrial and Engineering Chemistry, 15, 743-747, 2009.

[12] H. Ono, Y. Zhang, T. Yamada, Dissolving behavior and fate of cellulose in phenol liquefaction, Transactions of the Materials Research Society of Japan, 26, 807-812, 2001.

[13] W. Deng, M. Liu, Q. Zhang, Y. Wang, Direct transformation of cellulose into methyl and ethyl glucosides in methanol and ethanol media catalyzed by heteropolyacids, Catalysis Today, 164, 461-466, 2011. 